\title{
IMPACTO DE LA LUZ Y LA VENTILACIÓN NATURAL EN EL AMBIENTE LABORAL SOBRE EL SÍNDROME DEL EDIFICIO ENFERMO $Y$ LA PRODUCTIVIDAD
}

\author{
AUTORES: Valeria Pacheco Ochoa ${ }^{1}$ \\ Ana Lucía Jiménez Pérez ${ }^{2}$ \\ José Felipe Ramírez Pérez ${ }^{3}$
}

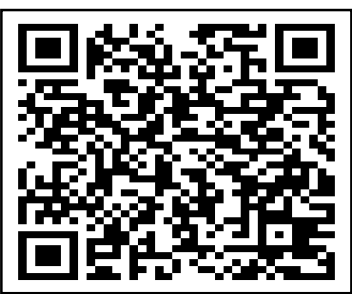

DIRECCIÓN PARA CORRESPONDENCIA: valeria.pacheco@uabc.edu.mx

Fecha de recepción: 12/01/2021

Fecha de aceptación: 28/06/2021

\section{RESUMEN}

El trabajo es una parte importante de la vida ya que permite la obtención de ingresos económicos. Asimismo, tener un trabajo satisfactorio es bueno para la salud física y mental de las personas. La Organización Mundial de la Salud en 1982 reconoció un síndrome el cual padecen los trabajadores, debido a las malas condiciones de la calidad del aire y del ambiente al interior de las construcciones. A este síndrome se le conoce como el Síndrome del Edificio Enfermo, el cual se utiliza para describir situaciones en las que los ocupantes experimentan problemas de salud físicos, mentales y molestias que parecen estar relacionadas con el tiempo que pasan en un edificio. Dicha situación afecta el desempeño laboral, la productividad y la salud del trabajador. El objetivo de la investigación es analizar el impacto que tiene la luz y ventilación natural en el ambiente de trabajo sobre el Síndrome del Edificio Enfermo y la productividad. La investigación tiene un enfoque cualitativo, con alcance exploratorio y diseño no experimental de corte transversal. Los resultados preliminares obtenidos evidencian que las malas condiciones físicas de trabajo afectan la salud física y psicológica del trabajador y lo exponen a condiciones de estrés laboral. Adicionalmente, es importante prestar atención a los factores internos y externos que impactan en los trabajadores, pues repercuten en su productividad y en su salud física y mental, tales son los casos de la luz y la ventilación natural, para evitar síntomas del Síndrome del Edificio Enfermo.

\footnotetext{
1 Arquitecta. Departamento de Servicios Administrativos. Universidad Autónoma de Baja California, Unidad Ensenada. Ensenada, Baja California, México. E-mail: valeria.pacheco@uabc.edu.mx

${ }^{2}$ Doctora en Ciencias Psicológicas. Facultad de Ciencias Administrativas y Sociales. Universidad Autónoma de Baja California, Unidad Valle Dorado. Ensenada, Baja California, México. E-mail: ana.jimenez14@uabc.edu.mx

${ }^{3}$ Ingeniero en Ciencias Informáticas. Facultad de Ciencias Administrativas y Sociales. Universidad Autónoma de Baja California, Unidad Valle Dorado. Ensenada, Baja California, México. E-mail: jramirez14@uabc.edu.mx
} 
Valeria Pacheco Ochoa, Ana Lucía Jiménez Pérez, José Felipe Ramírez Pérez

PALABRAS CLAVE: ambiente laboral; luz natural; productividad; síndrome del edificio enfermo; ventilación natural.

\title{
IMPACT OF NATURAL LIGHT AND VENTILATION IN THE WORK ENVIRONMENT ON SICK BUILDING SYNDROME AND PRODUCTIVITY
}

\begin{abstract}
Work is an important part of life because it provides economic income. Also, having a satisfying job is good for people's physical and mental health. In 1982, the World Health Organization recognized a syndrome suffered by workers due to poor air quality and environmental conditions inside buildings. This syndrome is known as Sick Building Syndrome, which it is used to describe situations in which occupants experience physical and mental health problems and discomfort that seem to be related to the time they spend in a building. This situation affects work performance, productivity and worker health. The aims is to analyze the impact of natural light and ventilation in the work environment on Sick Building Syndrome and productivity. The research has a qualitative approach, with exploratory scope and non-experimental cross-sectional design. The preliminary results obtained show that poor physical working conditions affect the physical and psychological health of workers and expose them to stressful working conditions. Additionally, it is important to pay attention to internal and external factors that impact on workers, as they affect their productivity and their physical and mental health, such as light and natural ventilation, in order to avoid symptoms of Sick Building Syndrome.
\end{abstract}

KEYWORDS: natural light; natural ventilation; productivity; sick building syndrome; work environment.

\section{INTRODUCCIÓN}

La Organización Mundial de la Salud (OMS) describe el Síndrome del Edificio Enfermo (SEE) como el conjunto de enfermedades y molestias que perciben los ocupantes de al menos el $30 \%$ de los edificios modernos y que afectan la salud física y mental de aproximadamente el $20 \%$ de los mismos, con un grupo de síntomas diversos (WHO, 1982). La US Environmental Protection Agency (EPA) en 1989 enfatizó que uno de los factores más importantes en la calidad de vida de los trabajadores es la estancia dentro de los edificios, pues pasan entre un 80 y $90 \%$ del tiempo en espacios cerrados, por lo que la luz y la ventilación natural son factores relevantes (EPA, 1989).

La luz y la ventilación natural en el espacio de trabajo son fundamentales para obtener una adecuada calidad del ambiente laboral. Cuando ambos factores se presentan de manera óptima, la calidad de vida del trabajador es buena, por lo que la productividad también aumenta (Taylor, Wakaf, Vasudevan \& Foster, 2017). En este sentido, el aprovechamiento de la luz natural tiene una elevada influencia sobre la salud mental y la productividad, si bien ayudan con la regulación del ritmo circadiano (Jagannath, Taylor, Wakaf, Vasudevan \& Foster, 2017; Sun, Hou, Cheng, Sheng, Zhang \& Sundell, 2019).

Al no contar con la luz natural adecuada, se puede dar una señal errónea al sistema, que afectaría el rendimiento laboral y provocaría problemas psicosociales en el trabajador. Los factores psicosociales como el estrés laboral influyen en los síntomas del SEE. Del mismo modo, se sabe

98 UNESUM-Ciencias. Publicación cuatrimestral. Vol. 5, Año 2021, No. 4 (Número Especial) 
que muchos factores de construcción influyen en estos síntomas, incluido el tipo ventilación, la tasa de ventilación del aire exterior, la temperatura y la humedad interior (Fisk, 2000; Ghaffarianhoseini et al., 2018).

Jansz (2017) desarrolló un modelo de causas del SEE, el cual resalta los factores que influyen para padecer los síntomas del SEE. Estos factores son bilógicos, químicos, físicos, ergonómicos, de ventilación, de diseño de construcción y de materiales de construcción. Jansz señala que estos factores presentes en malas condiciones pueden provocar severos daños a la salud. En la Figura 1 se visualiza el modelo desarrollado por Jansz (2017), donde se describen las causas del SEE:

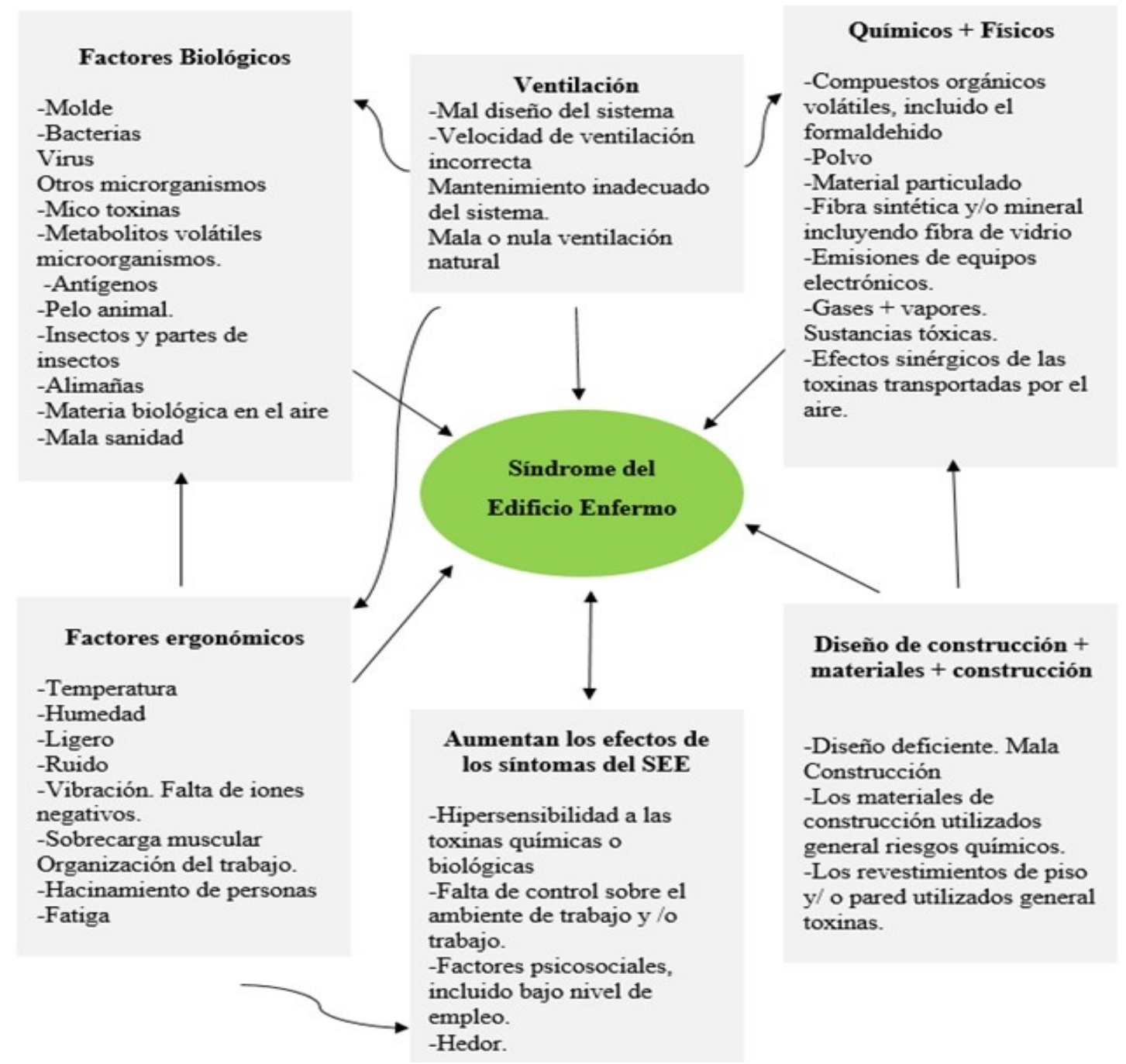

Figura 1. Modelo de causas del SEE. Fuente: (Jansz, 2017).

Por medio del SEE se obtienen indicadores de salud del trabajador y comportamientos de riesgo asociados al espacio de trabajo (Jansz, 2017). Teniendo como antecedente que la luz y la ventilación natural son importantes para lograr un desempeño laboral favorable, es importante 
evaluar los factores psicosociales asociados a la vida laboral que impactan sobre el trabajador por la deficiencia de luz y ventilación natural en sus espacios de trabajo (Nakayama et al., 2019).

\section{ANTECEDENTES}

La salud física y mental de los trabajadores constituye una prioridad para los empresarios y patrones, por lo que se deben fomentar entornos laborales favorables y políticas de prevención y atención a los riesgos psicosociales en los centros de trabajo (OMS, 2019). Vargas (2005) hace referencia al documento emitido por el Instituto Nacional de Seguridad e Higiene en el trabajo en España en el 2010, el cual señala que los problemas de calidad de ambiente interior como la iluminación y la ventilación natural provocan problemas de salud como: dolores de cabeza, irritación, congestión, fatiga y vista cansada, también conocidos como SEE. La salud ambiental es la disciplina de la Salud Pública que identifica, caracteriza, vigila, controla y evalúa los efectos de los riesgos físicos, químicos, psicosociales y biológicos del medio ambiente sobre la salud humana, por lo que es importante cuidar la calidad del ambiente del interior donde se labora (Ríos-Aceves, Macías-Hernández, Ventura-Houle \& Baez, 2020).

Derivado de esta perspectiva surge el concepto de SEE. Es una expresión que se utiliza para describir situaciones en las que los ocupantes de los edificios experimentan efectos nocivos agudos sobre su salud, así como falta de confort asociado al tiempo de permanencia en estos lugares. En cuanto a salud mental, es la forma en que pensamos y sentimos, y nuestra capacidad para lidiar con los problemas. Cuando se disfruta de una buena salud mental, se tiene un sentido de propósito y dirección, la energía para hacer las cosas que se quiere hacer y la capacidad de lidiar con los desafíos que se presentan en la vida. Cuando se disfruta de una buena salud mental se puede aprovechar al máximo el potencial. No obstante, la salud mental no siempre es la misma, puede fluctuar por las circunstancias. En tal sentido, los efectos adversos producidos por el SEE obligan la medicación para su solución, provocando nuevos problemas para las personas como para la sociedad (Oswal, 2015; Zayas, Pérez \& Pérez, 2020).

Warr (1992) reportó evidencia de que las malas condiciones físicas de trabajo incrementan el estrés sufrido por los trabajadores a su salud física y psicológica. En su estudio, aplicado a 600 trabajadores de la Universidad de Port Harcourt (UNIPORT), identificó cinco riesgos principales, los cuales fueron riesgos ergonómicos $(72,2 \%)$, calor $(66,6 \%)$, exceso de trabajo $(66,6 \%)$, mala ventilación (54,8\%) y exposición a sustancias químicas (50,8\%). Las enfermedades más comunes de las que se informó eran dolor de espalda (56\%), dolor de cabeza $(53,2 \%)$ y fatiga/debilidad (53,2\%). Adicionalmente se evidenció una relación fuerte entre factores relacionados con el trabajo, enfermedades profesionales y problemas psicosociales.

La investigación de Tuaycharoen \& Tregenza (2007) aborda la luz natural como factor ambiental. En los experimentos realizados se evaluó la cantidad de luz natural hacia el interior y el deslumbramiento de las ventanas de un edificio de 20 pisos en Sheffield, Reino Unido. Las oficinas seleccionadas fueron idénticas, excepto que en diferentes niveles y orientaciones, las perspectivas variaban de cerrar vistas de otros edificios a extensos paisajes. Los resultados arrojaron que para el segundo caso hubo una mayor productividad y creatividad debido a la conexión obtenida con el exterior, su incidencia sobre el reloj biológico y el ritmo circadiano. 
En la Figura 2 se muestra la recomendación ideal para colocar una ventana en invierno o verano. La flecha roja representa la dirección del sol en verano y la línea amarilla en invierno, cómo ingresa a una oficina y en qué ángulo. La sección A ejemplifica la peor opción, mientras que la sección C es la mejor. En esta última sección se agrega un elemento arquitectónico a la ventana que permite evitar el deslumbramiento en el trabajador por la incidencia del sol.
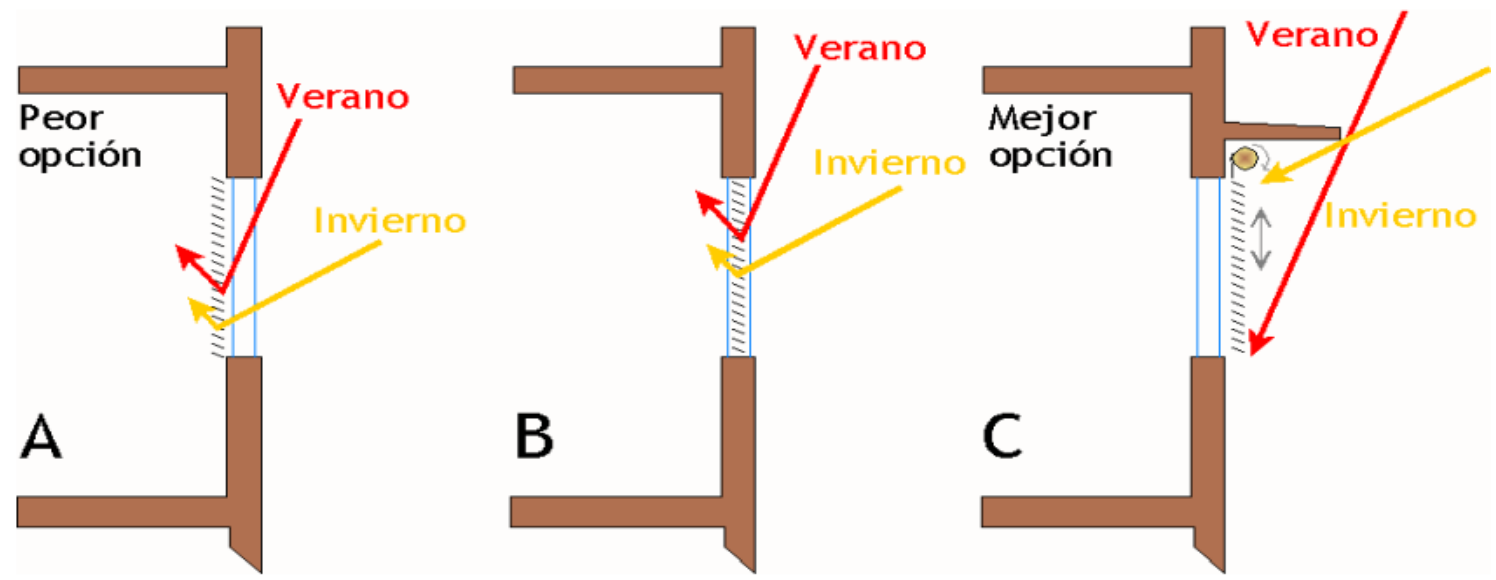

Figura 2. Recomendación de ventanas en verano o invierno. Fuente: (Sánchez \& Montañés, 2020).

La importancia de los factores externos como los estímulos lumínicos o ciertos comportamientos para la regulación del ritmo circadiano, como dormir por la noche y estar despierto durante el día, son ejemplos de un ritmo circadiano relacionado con la luz natural. De ahí radica la importancia de la luz natural para las actividades en el ser humano, en este caso para el trabajador dentro de las oficinas, ya que se puede dar una señal errónea al cuerpo humano y este afectaría el rendimiento laboral, provocando problemas psicosociales en el trabajador (Deschères, 2015).

Newsham, Aries, Mancini \& Faye (2008) en su investigación determinaron que las personas que trabajan con luz natural en su oficina presentan menos problemas de salud y estrés que los trabajadores que pasan su jornada laboral en un espacio de trabajo sin ventanas, sin luz y sin ventilación natural. No obstante, el exceso de ventanas en un espacio de trabajo también provoca falta de productividad, debido al aumento excesivo de la temperatura (Carrillo \& Villadiego, 2019). Por todo ello, para el diseño de un espacio de trabajo es conveniente considerar la forma y tamaño de las ventanas para lograr un óptimo ambiente de confort al interior y así aportar a la productividad del trabajador. Para Jansz (2017) el diseño de los edificios y los materiales que se utilizan al interior de los mismos son las principales causas del SEE. Los contaminantes que se pueden generar al interior de una ofician por falta de luz y ventilación natural, como presencia de hongos, son nocivos para la salud. También hay factores que mejoran los efectos de SEE que están influenciados por el manejo organizacional, factores personales y factores psicosociales.

En España, la Institución Nacional de Seguridad e Higiene en el Trabajo (INSHT) cuenta con la norma NTP-290: El síndrome del edificio enfermo, cuestionario para su detección. Este cuestionario permite diagnosticar si el personal padece el SEE, basándose en el porcentaje permitido por la OMS no mayor al 20\% (Solé \& Pérez, 1991). El trabajo es beneficioso para la 
salud mental, sin embargo, un entorno laboral negativo puede causar problemas. La OMS en mayo del 2019 publicó un artículo titulado "Salud mental en el lugar de trabajo" en el cual menciona que la depresión y la ansiedad tienen repercusiones económicas importantes y que se estima que cuesta anualmente a la economía mundial US\$ 1 billón en pérdida de productividad. Las organizaciones pueden aplicar muchas medidas eficaces para promover la salud mental en el lugar de trabajo y aumentar con ello la productividad (OMS, 2019). El citado estudio de la OMS reporta que por cada US\$ 1 invertido en la extensión del tratamiento de los trastornos mentales frecuentes se obtiene un rendimiento de US\$ 4 en mejora de la salud y la productividad. En el mundo hay 264 millones de personas que padecen depresión, una de las principales causas de discapacidad y ansiedad. Esto afecta directamente la productividad. Los problemas de depresión y ansiedad están relacionados al ambiente de trabajo, por la falta de luz y ventilación natural.

La productividad se define como el resultado de las acciones que se llevan a cabo para conseguir los objetivos de la empresa, teniendo en cuenta la relación entre los recursos que se invierten y los resultados de los mismos (Kersemaekers et al., 2018). En la productividad laboral y para la obtención de ventajas competitivas sostenidas es importante gestionar el entorno de una organización en diversas dimensiones: entorno ambiental, ambiente físico, entorno psicosocial y de relaciones sociales, las cuales posibilitan la obtención de un lugar de trabajo con mejor calidad de vida, saludable y sostenible (Johnson, Zimmermann \& Bird, 2019; Pérez, López-Torres \& Morejón-Valdés, 2021). Otros autores agregan que un entorno saludable es esencial para aumentar la productividad, la motivación, la satisfacción laboral y la calidad de vida de los trabajadores (Demaría, Farías \& Acevedo, 2017; Segredo, 2013).

Por todo lo abordado, diversos autores afirman que el diseño interior es importante para los trabajadores que laboran dentro de una oficina, ya que las condiciones ambientales, estéticas y de confort actúan en el apropiado desarrollo de actividades y en la productividad. La iluminación y la ventilación natural fomentan la productividad en el lugar de trabajo. Adicionalmente, la luz y la ventilación natural deben ser cuidadosamente controladas de forma que se eviten niveles incómodos de claridad, deslumbramiento o aumento de la temperatura. La altura de recorrido del sol cambia a lo largo del año, está en su ángulo más bajo en invierno y en el más alto en verano. La orientación Sur es constante y la Norte es difusa, pero es la más conveniente para trabajar con computadoras, ya que minimiza los potenciales reflejos en los monitores. La orientación Oeste provoca la incidencia del sol por las tardes, mientras que la orientación Este favorece la presencia del sol en las mañanas. (Grimley \& Love, 2012).

Las organizaciones deben tratar de que sus empleados se encuentren satisfechos y cómodos en sus áreas de trabajo. Para ello, han de procurar que laboren en un entorno físico adecuado y con condiciones favorables. Los sitios ruidosos, los lugares calurosos y las áreas congestionadas o mal ventiladas perjudican al trabajador y afectan su rendimiento (Vargas, 2017). En este sentido, la productividad está ligada al entorno laboral, las condiciones físicas en las que se encuentra el área de trabajo, el clima laboral y el entorno físico.

Para obtener un espacio físico favorable para el colaborador se debe apoyar en normas y reglamentos de construcción, así como en normativas para identificar los riesgos psicosociales, para poder cumplir con ellos y a su vez dar satisfacción al colaborador de laborar en un espacio agradable, que lo inspire y aporte a su productividad. En la productividad laboral es importante gestionar el entorno de trabajo de una organización en diversas dimensiones: absentismo por 
problemas de salud, decisiones, carga de trabajo, falta de control, demanda de tareas, cumplimiento de tareas, estrés y esfuerzo cognitivo, las cuales posibilitan alcanzar un lugar de trabajo con mejor calidad de vida, saludable y sostenible (Cano, 2017).

\section{MÉTODO}

El objetivo del presente trabajo es analizar el impacto que tiene la luz y ventilación natural en el ambiente de trabajo sobre el Síndrome del Edificio Enfermo y la productividad. La investigación tiene un enfoque cualitativo, con alcance exploratorio y diseño no experimental de corte transversal. Los principales métodos científicos aplicados fueron:

- $\quad$ El análisis documental: Se aplica como técnica de recolección de datos para el estudio de los referentes teóricos de la investigación, con apoyo en la consulta de libros y artículos científicos, con predominio del 61\% de bibliografía en los últimos 5 años (2017-2021).

- Método analítico-sintético: Permite el estudio de los principales variables de la investigación, las cuales son el ambiente laboral, la luz natural, la ventilación natural, la productividad y el SEE, posibilitando un análisis de lo general a lo particular y luego una síntesis de lo simple a lo complejo, para la determinación de conclusiones.

El estudio fue realizado entre octubre de 2020 y mayo de 2021, en Ensenada, Baja California, México. Se centra en la investigación del SEE y su impacto sobre la productividad y el rendimiento laboral. Este síndrome es reconocido como enfermedad por la OMS desde 1982.

\section{RESULTADOS Y DISCUSIÓN}

Con base en la literatura científica analizada, los resultados exploratorios que se presentan en el trabajo permiten realizar un acercamiento a las principales variables y dimensiones asociadas con el SEE y cómo este impacta negativamente sobre la productividad laboral, si bien esta enfermedad silenciosa cada año afecta considerablemente a las organizaciones y su adecuado desempeño. Los resultados presentados se soportan en referencias actualizadas y especializadas en la temática. En la Figura 3 se muestra una representación de las variables y dimensiones del fenómeno de estudio. 


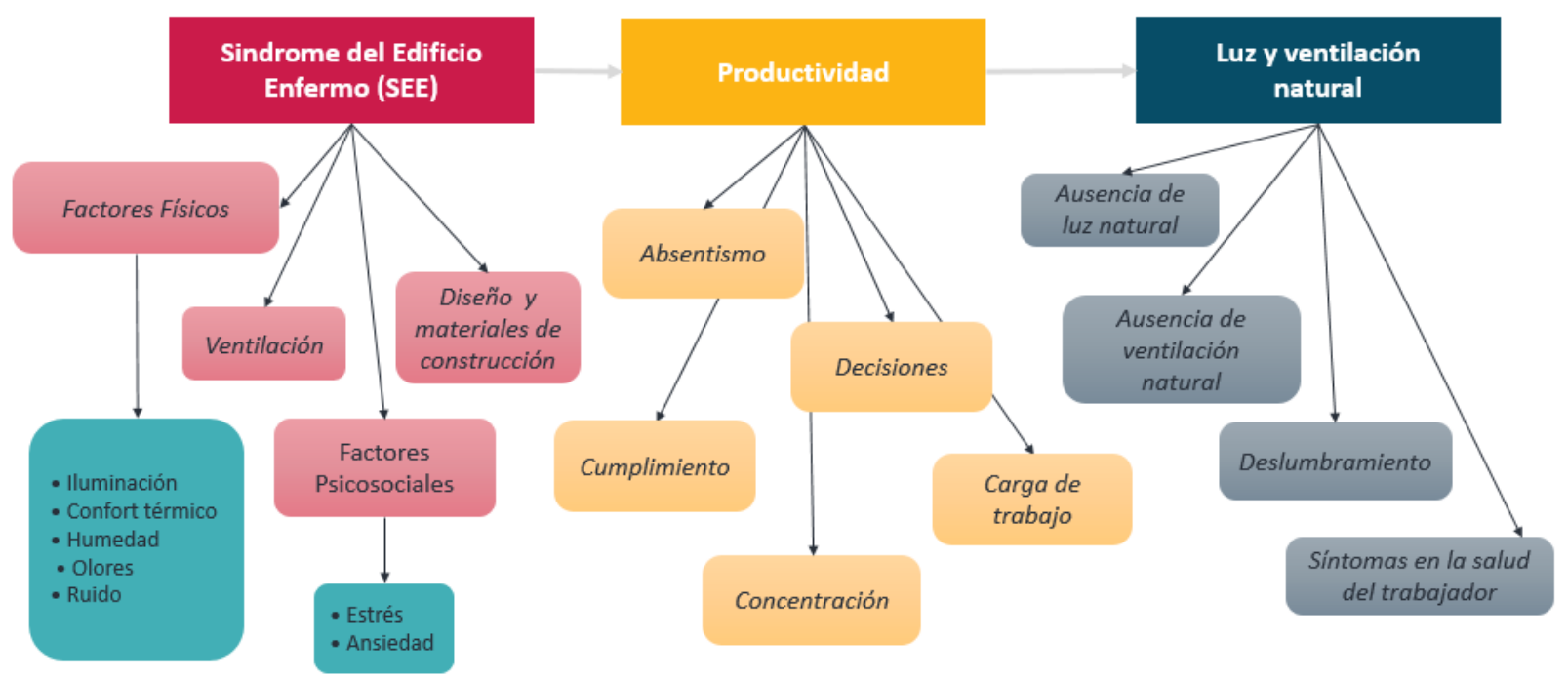

Figura 3. Representación de variables y dimensiones relacionadas con el SEE. Fuente: elaboración propia.

El SEE es un factor que influye en el rendimiento laboral, pues afecta la salud física y mental del trabajador. Al respecto, Sánchez \& Therán (2019) realizaron una investigación en Colombia, en la Facultad de Educación y Ciencias Humanas de la Universidad de Córdoba. En la misma se estudió el SEE, donde se implementó un estudio en 34 funcionarios académicos, a partir de un muestreo intencional. Se les aplicó el instrumento NTP-290 Detección de Síndrome del Edifico Enfermo, para determinar factores de riesgo en el trabajador. Se determinó la existencia de SEE debido a que se superó el índice de 20\% propuesto por la OMS en 1982.

En los resultados predominó la presencia de factores físicos negativos como mucho calor o frío, existencia de ruido, falta de ventilación, estancamiento del aire, ventanas que no se podían abrir o la lejanía a estas. De igual manera, se encontraron factores psicosociales que afectaban el desempeño como el estrés. Atendiendo a tales situaciones, se describieron los síntomas predominantes en los funcionarios académicos, como la sequedad en los ojos, una visión borrosa, estornudos, tos, sequedad en la boca, sensación de sed, sequedad en la piel, reflujo, estreñimiento, dolores de espalda, dolores musculares, dolores en las articulaciones, debilidad, ansiedad, agotamiento, dolor de cabeza, aletargamiento y falta de energía (Sánchez \& Therán, 2019).

En otro estudio, realizado por Terán \& Haro (2015) a tres edificaciones de la Facultad de Ciencias de la Ingeniería de la Universidad Tecnológica Equinoccial de Quito en Ecuador, se detectó falta de confort como resultado de contar con puestos de trabajo muy alejados o muy próximos a ventanas, activándose el indicador del SEE. La molestia de no tener luz natural o en exceso, así como la necesidad de contar con zonas de calor natural producido por los rayos solares, inducen a los trabajadores a salir a lugares de mayor temperatura en determinados momentos de su labor. Los resultados aquí discutidos son similares a los obtenidos por Morán, Yábar \& Figueroa (2017) y Zuo, Luo \& Liu (2021).

En consonancia con los resultados antes discutidos, los cuales se corresponden consistentemente con los presentados en la investigación, Erazo, Huertas \& Jojoa (2016) realizaron una revisión documental sobre el comportamiento del SEE en los trabajadores a nivel mundial durante el

104 UNESUM-Ciencias. Publicación cuatrimestral. Vol. 5, Año 2021, No. 4 (Número Especial) 
período comprendido de 2000 a 2014. El estudio reportó la presencia de enfermedades cuyo origen se derivaba del desarrollo de la actividad laboral en edificios herméticos o con sistemas centralizados de control de la ventilación y aire acondicionado. En el artículo se arribó a la conclusión de que en todos los estudios analizados existieron sintomatologías asociadas con la presencia del SEE (Erazo et al., 2016). Lu et al. (2018) arriba a similares conclusiones e igualmente se corresponden con los factores, variables y dimensiones presentadas en la investigación.

En México, un estudio realizado por Ríos-Aceves et al. (2020) en una Dependencia de Educación Superior (DES) dentro del Centro Universitario de Ciudad Victoria en Tamaulipas, tuvo como objetivo evaluar el SEE de acuerdo con los espacios y entorno de trabajo de los ocupantes e identificar la presencia de síntomas relacionados con el SEE en los usuarios que permitan generar un diagnóstico sobre el nivel de salud organizacional. El estudio estuvo conformado por dos edificios que albergan personal docente y administrativo, con un diseño de corte transversal aplicando un método cuantitativo, por medio de un cuestionario como herramienta para colectar los datos, para identificar los posibles síntomas presentes de SEE, en un espacio y tiempo determinado. Como resultado se obtuvo que los Profesores de Tiempo Completo son los que presentaron mayor cantidad de síntomas en comparación con los Profesores de Asignatura. Se determinó que el $20 \%$ del total de los trabajadores padecen síntomas que se relacionan con el SEE (Ríos-Aceves et al., 2020).

Asimismo, en la Universidad Autónoma de Nuevo León, en la Facultad de Psicología, Pereyra (2016) diseñó un plan de intervención para la disminución del estrés laboral en una empresa gubernamental. Se aplicó el Inventario de Maslach Burnout a los empleados. Dicha escala es la más utilizada a nivel mundial para la medición del Estrés Laboral, el cual está estrechamente relacionado con las sintomatologías del SEE. Los resultados confirmaron la existencia de estrés laboral y la presencia del SEE, determinándose la ocurrencia de diversos problemas mentales como adicciones, ansiedad, problemas de personalidad, depresión y problemas sicóticos (Pereyra, 2016).

A todo esto, en México no se cuenta con una normatividad que brinde una guía o una metodología para determinar el nivel de SEE, por lo que se utiliza la normatividad promulgada en España, Estados Unidos o Reino Unido (Ríos-Aceves et al., 2020). En el 2017 se realizó una investigación en el hospital de gineco-pediatría (HGP/MF) $\mathrm{N}^{\circ} 31$ de Instituto Mexicano del Seguro Social (IMSS) en Mexicali, Baja California. La misma se enfocó en la identificación de sintomatologías causadas por el SEE mediante la aplicación del cuestionario NTP-290. Se realizó un estudio observacional-descriptivo, transversal, unicéntrico. En el mismo se observó que de la sintomatología interrogada, seis de los ocho síntomas estudiados (oculares, nasales, garganta, dolor de cabeza, síntomas parecidos a la gripe, trastornos cutáneos, trastornos respiratorios, estrés) fueron referidos por los trabajadores en porcentajes mayores al 20\%. Los autores concluyeron que se debe catalogar el edificio estudiado como portador del SEE (Lizcano, Palmer, Quiñones, Medina \& López, 2017). A la fecha no se han encontrado más evidencias empíricas publicadas que presenten o describan tal fenómeno en la región. 


\section{CONCLUSIONES}

Con el análisis de la información recopilada se llega a la conclusión de que es importante prestar atención en los factores internos y externos que impactan en los trabajadores, ya que repercuten en su productividad y en su salud física y mental. Principalmente los factores iluminación y ventilación natural se han convertido en una excelente herramienta para mejorar la productividad laboral, ya que está asociada de forma ineludible con el ser humano, al igual que con las plantas, aportando bienestar. La ausencia de iluminación y ventilación natural en áreas de trabajo, donde la mayoría de las personas pasan más tiempo que en sus propios hogares, se demuestra que repercute negativamente en la eficiencia y en la salud de los trabajadores. Adicionalmente, ambas variables son determinantes para el bienestar del trabajador, su estado de ánimo y su salud.

Por todo ello, una adecuada ubicación del área de trabajo y la orientación de ventanas (Norte, Sur, Este, Oeste), es fundamental para una adecuada iluminación y ventilación natural. Atendiendo a ello se pudiera mejorar el ambiente (temperatura) físico al interior de las áreas de trabajo, probando sensaciones de agrado, aumentando el estado de ánimo y evitando situaciones de estrés, pérdida de concentración y un ahorro de energía eléctrica.

En la región de Baja California son pocas las investigaciones sobre el SEE, identificándose hasta el momento solo un artículo relacionado con el tema en la ciudad de Mexicali. En cambio, en España y en Colombia es en donde se han encontrado más contribuciones sobre esta problemática y su relación con la productividad y la salud del trabajador. Adicionalmente, los autores consideran que se debe investigar más sobre el impacto que tiene la luz y la ventilación natural sobre la productividad en la región y en México.

La investigación realizada tuvo un alcance exploratorio, es por ello que los trabajos futuros se deben dirigir a analizar puntualmente, por medio de intervenciones, el impacto que tiene la luz y la ventilación natural en el ambiente laboral sobre el Síndrome del Edificio Enfermo y la productividad.

\section{REFERENCIAS BIBLIOGRÁFICAS}

Carrillo, M. P. F., \& Villadiego, M. J. O. (2019). Síndrome del Edificio Enfermo en la facultad Ciencias de la Salud de la Universidad de Córdoba $2019 . \quad$ Recuperado de https://repositorio.unicordoba.edu.co/handle/ucordoba/2412

Demaría, M. J., Farías, M. A., \& Acevedo, G. E. (2017). Factores de riesgos psicosociales presentes en el ambiente de trabajo de Enfermería en hospitales públicos de Córdoba, Jujuy, La Pampa y Santiago del Estero, Argentina. Revista de Salud Pública, 60-72.

Deschères, L. (2015). Los efectos visuales y biológicos de la Luz. Revista de iluminación Iluminet. Recuperado de https://www.iluminet.com/los-efectos-visuales-y-biologicos-de-la-luz/

Erazo, B; Huertas, D \& Jojoa, L. (2016). Revisión bibliográfica relacionada con el comportamiento del Síndrome del Edificio Enfermo en los trabajadores a nivel mundial durante el período 2000 - 2014. Trabajo de investigación Universidad CES. Facultad de Medicina. Especialización en Gerencia de la Seguridad y Salud en el Trabajo. San Juan de Pasto, Colombia. Recuperado de http://repository.ces.edu.co/handle/10946/1679

Fisk, W. J. (2000). Health and productivity gains from better indoor environments and their relationship with building energy efficiency. Annual review of energy and the environment, 25(1), 537-566. 
Ghaffarianhoseini, A., AlWaer, H., Omrany, H., Ghaffarianhoseini, A., Alalouch, C., Clements-Croome, D., \& Tookey, J. (2018). Sick building syndrome: are we doing enough?. Architectural Science Review, 61(3), 99121.

Jagannath, A., Taylor, L., Wakaf, Z., Vasudevan, S. R., \& Foster, R. G. (2017). The genetics of circadian rhythms, sleep and health. Human molecular genetics, 26(R2), R128-R138.

Jansz, J. (2017). In Quah S. R. (Ed.), Sick building syndrome. Oxford: Academic Press.

Johnson, B., Zimmermann, T., \& Bird, C. (2019). The effect of work environments on productivity and satisfaction of software engineers. IEEE Transactions on Software Engineering.

Kersemaekers, W., Rupprecht, S., Wittmann, M., Tamdjidi, C., Falke, P., Donders, R. \& Kohls, N. (2018). A workplace mindfulness intervention may be associated with improved psychological well-being and productivity. A preliminary field study in a company setting. Frontiers in psychology, 9, 195.

Lizcano, J., Palmer, L., Quiñones, K., Medina, M., \& López, D. (2017). Sintomatología causada por el síndrome del edificio enfermo en trabajadores de la salud en ambiente hospitalario. Revista cubana de salud y trabajo, 18, pp. 52-56.

Lu, C. Y., Tsai, M. C., Muo, C. H., Kuo, Y. H., Sung, F. C., \& Wu, C. C. (2018). Personal, psychosocial and environmental factors related to sick building syndrome in official employees of Taiwan. International journal of environmental research and public health, 15(1), 7.

Morán, L., Yábar, G., \& Figueroa, K. (2017). Calidad del aire interior en el síndrome del edificio enfermo, ciudad de Trujillo. Revista de la Facultad de Medicina Humana, 17(4).

Nakayama, Y., Nakaoka, H., Suzuki, N., Tsumura, K., Hanazato, M., Todaka, E., \& Mori, C. (2019). Prevalence and risk factors of pre-sick building syndrome: characteristics of indoor environmental and individual factors. Environmental health and preventive medicine, 24(1), 1-10.

Newsham, G. R., Aries, M. B. C., Mancini, S. M., \& Faye, G. (2008). Individual control of electric lighting in a daylit space. Lighting Research and Technology, 40(1), 2541.

OMS. (2019). Salud mental en el lugar de trabajo. Hoja informativa. Portal oficial de la Organización Mundial de la Salud (OMS). Disponible en: https://www.who.int/mental_health/in_the_workplace/es/

Pereyra, C. M. (2016). Diseño de un plan de intervención para la disminución de estrés laboral en una empresa gubernamental .Tesis Doctoral, Universidad Autónoma de Nuevo León. México.

Pérez, J. F. R., López-Torres, V. G., \& Morejón-Valdés, M. (2021). Information and Communication Technologies as a competitive performance factor in provider institutions of medical services in Ensenada, Baja California. Journal of Administrative Science, 2(4), 31-37.

Ríos-Aceves, K. E., Macías-Hernández, B. A., Ventura-Houle, R., \& Baez, A. A. (2020). Salud ambiental en interiores: edificios enfermos. Sociedad y Ambiente, (22), 1-21.

Sánchez, J. J. Á, \& Therán, A. M. M. (2019). Síndrome del Edificio enfermo en la Facultad de Educación y Ciencias Humanas de la Universidad de Córdoba, periodo 2019-II. Recuperado de https://core.ac.uk/download/pdf/286767274.pdf

Segredo, A. (2013). “Clima organizacional en la gestión del cambio para el desarrollo de la organización”. Revista cubana de salud pública, 39(2), pp. 385-393.

Solé, M. D., \& Pérez, J. (1991). El síndrome del edificio enfermo: cuestionario para su detección NTP-290. Barcelona, INSHT. Recuperado de https:/www.insst.es/documents/94886/327166/2_ntp_290.pdf/30db9f6fd401-4f69-929d-e735cfaf5855

Sun, Y., Hou, J., Cheng, R., Sheng, Y., Zhang, X., \& Sundell, J. (2019). Indoor air quality, ventilation and their associations with sick building syndrome in Chinese homes. Energy and Buildings, 197, 112-119. 
Terán, F. O., \& Haro, B. H. (2015). Sick building syndrome in blocks of an academic institution of higher education. Enfoque UTE, 6(2), 15-24.

Tuaycharoen, N., \& Tregenza, P. R. (2007). View and discomfort glare from windows. Lighting Research \& Technology, 39(2), 185-200.

US Environmental Protection Agency (1989). Report to Congress on indoor air quality, volume II: assessment and control of indoor air pollution. Technical Report EPA/400/1-89/001C.

Vargas, A. M. J. (2017). Importancia del clima organizacional en la productividad laboral. Colombia. Recuperado de

http://biblioteca.esucomex.cl/RCA/Importancia\%20del\%20clima\%20organizacional\%20en\%20la\%20productividad \%20laboral.pdf

Warr, P.B. (1992). Job features and excessive stress. In R. Jenkins \& N. Coney (Eds.), Prevention of Mental Ill Health at Work. London: HMSO.

World Health Organization (WHO). (1982). Indoor Air Pollutants Exposure and Health Effects Report on a WHO Meeting Nördlingen, 8-11 June 1982. EURO reports and studies, 78.

Zayas, J. C. B., Pérez, J. F. R., \& Pérez, A. D. R. R. (2020). Interacciones medicamentosas como un problema de salud imperceptible en la población. Revista Cubana de Medicina General Integral, 36(1), 1-15.

Zuo, C., Luo, L., \& Liu, W. (2021). Effects of increased humidity on physiological responses, thermal comfort, perceived air quality, and Sick Building Syndrome symptoms at elevated indoor temperatures for subjects in a hot-humid climate. Indoor air, 31(2), 524-540.

108 UNESUM-Ciencias. Publicación cuatrimestral. Vol. 5, Año 2021, No. 4 (Número Especial) 